\title{
Base of skull fracture leading to pneumomediastinum and pneumo-retroperitoneum: a case report with review of literature
}

\author{
Garima Sharma $^{* \mathbb{D}}$, Sanya Vermani ${ }^{\mathbb{D}}$, Anjum Syed ${ }^{\mathbb{D}}$ \\ Department of Radiodiagnosis, All India Institute of Medical Sciences, Rishikesh, India
}

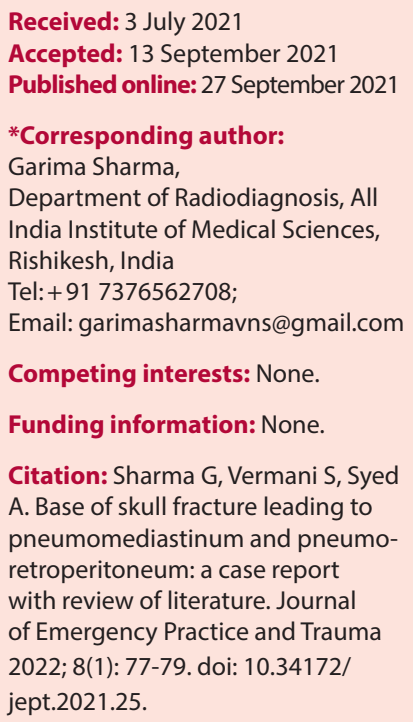

\begin{abstract}
Objective: The presence of air within the mediastinal compartment and retro-peritoneal compartment, in the setting of trauma, can be because of visceral and skeletal injuries. However, in absence of a local site injury, an approach based on anatomical communication between various body compartments should be utilized and all potential sites of injuries must be reviewed.

Case Presentation: We present a case of a 40 -year-old male patient with a history of trauma (fall from height), presenting to the emergency department with loss of consciousness and ear bleed. Chest radiographs showed pneumomediastinum. On cross-sectional imaging, pneumomediastinum and pneumoretroperitoneum were seen, however no esophageal, tracheal and skeletal injuries could be identified. On careful evaluation, fractures involving the base of skull were identified as a source of ectopic air.

Conclusion: This case represents a situation where the fascial connections between various compartments of the body were utilized to find the site of injury and hence the source of ectopic air. Base of skull fractures are important to be identified since these require surgical attention at an early stage.

Keywords: Trauma, Pneumomediastinum, Pneumo-retroperitoneum
\end{abstract}

\section{Introduction}

An anatomical continuum exists between the cervical, mediastinal, retroperitoneal, extraperitoneal abdomen and pelvic spaces, based on the embryologic development, allowing ectopic air to spread along the various compartments (1). Pneumomediastinum following trauma can occur due to various causes which include visceral injuries such as tracheal, oesophageal or skeletal injuries such as rib fractures. If none of these causes are present, we should make a search for the origin of ectopic air for which thorough anatomical knowledge is necessary. We report a case of traumatic base of skull fractures leading to pneumomediastinum and pneumo-retroperitoneum.

\section{Case Presentation}

A 40-year-old male presented to the emergency department of our institute with the history of fall from height of about 12 feet, loss of consciousness and ear bleed. On initial survey, the patient had low Glasgow Coma Score (E1V1M3) with compromised airway and falling oxygen saturation. Air entry was reduced in bilateral lungs. Focused assessment with sonography for trauma
(FAST) and pelvic compression tests were negative. Patient was intubated and given oxygen support. Cervical stabilization was done and intravenous fluids were started. Chest radiograph revealed pneumomediastinum (Figure 1). After stabilization, the patient underwent noncontrast-enhanced computed tomography (CT) of head and contrast-enhanced CT of thoracic and abdominal regions.

Non-contrast enhanced CT head showed hemorrhagic contusions in bilateral frontal lobes, subarachnoid haemorrhage in right fronto-parieto-temporal regions, subdural haemorrhage along with frontal convexity and intraventricular haemorrhage in the fourth ventricle. Few foci of air were seen along the cerebral convexity. Soft tissue scalp swelling was seen in the occipital region on the left side.

On bone window, mildly displaced fracture of occipital bone was seen, extending inferiorly to involve left lambdoid suture with sutural diastasis. Another linear undisplaced fracture of right partial bone was seen, extending inferiorly to the mastoid part of right temporal bone with resultant hemomastoid. Fracture of lateral mass 


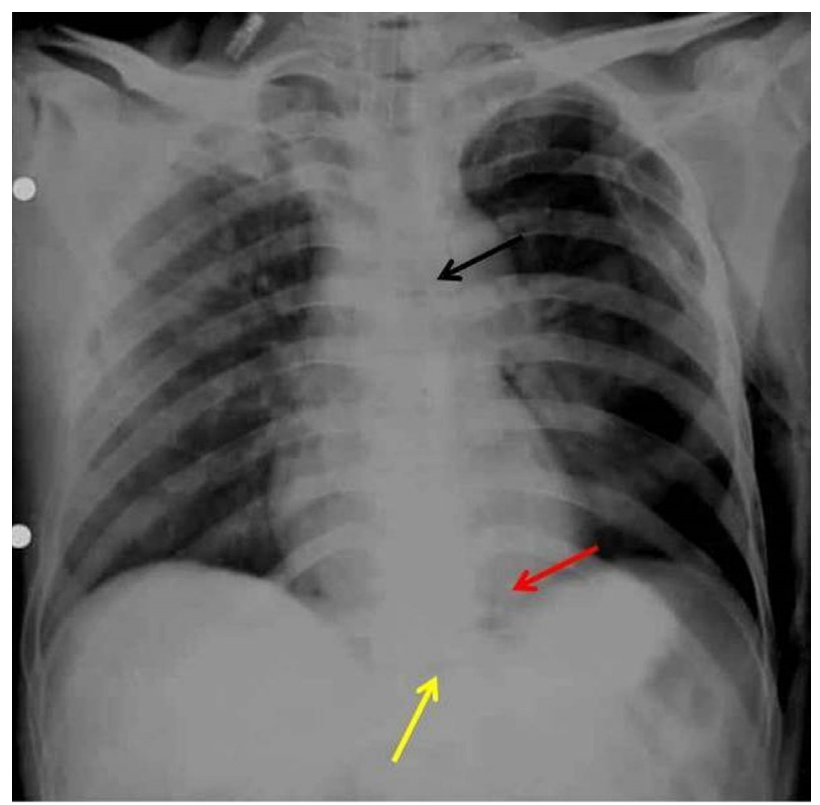

Figure 1. Frontal chest radiograph shows air outlining the mediastinal structures (black arrow). "V" shaped air collection is seen along the left lateral mediastinal border and left hemi diaphragm suggestive of "Naclerio V sign" (red arrow). Air is also seen beneath the central tendon of diaphragm (yellow arrow).

of $\mathrm{C} 1$ vertebra was seen on the right side. Also, fractures of the occipital condyle and posterior skull base were seen on the right side (Figure 2)

Fractures of sphenoid sinus walls along with hemosinus were seen. Also, fractures of posterolateral wall of bilateral maxillary sinuses were identified with associated hemosinus.

Contrast-enhanced CT of thorax and abdomen revealed pneumomediastinum with air extending along the subcutaneous planes of neck reaching upto the base of skull. Air space opacities were seen in dependent regions of bilateral lungs suggestive of aspiration. No pneumothorax was seen. No other skeletal injuries were identified elsewhere. Abdominal sections showed significant free air around pancreas and perihepatic space (Figure 3). There was no gross solid organ injury. No free fluid was seen on abdominal sections. CT Esophagogram was done to look for esophageal injury, which showed no contrast extravasation into the mediastinum.

Serial chest radiographs taken over 8 days showed no increase in pneumomediastinum or pneumoretroperitoneum.

On review and careful evaluation, the source of ectopic air was identified to be fractures involving the skull base.

In the absence of any airway or esophageal injury, rib or clavicular fractures or direct lung trauma, penetrating injury to the neck or chest, it was concluded that the source of ectopic was skull base fractures.

The patient was kept on the ventilator and managed with supportive care. Nine days later, the patient developed respiratory distress and suffered cardiac arrest.

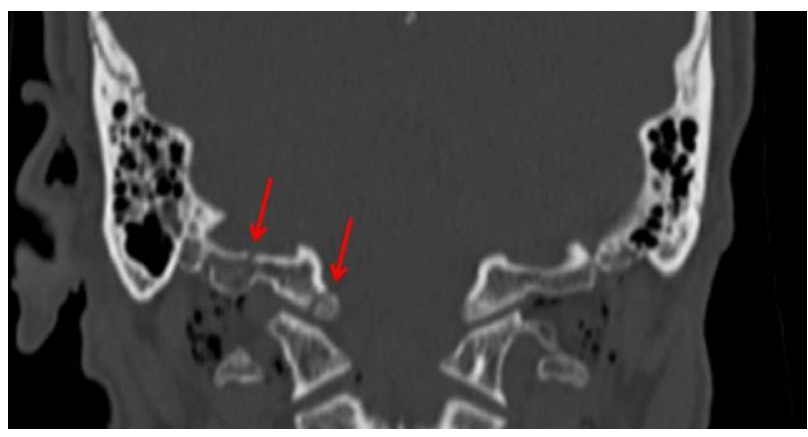

Figure 2. Non-contrast enhanced CT of the head coronal section in the bone window shows fracture of occipital condyle and posterior skull base on the right side (red arrows).

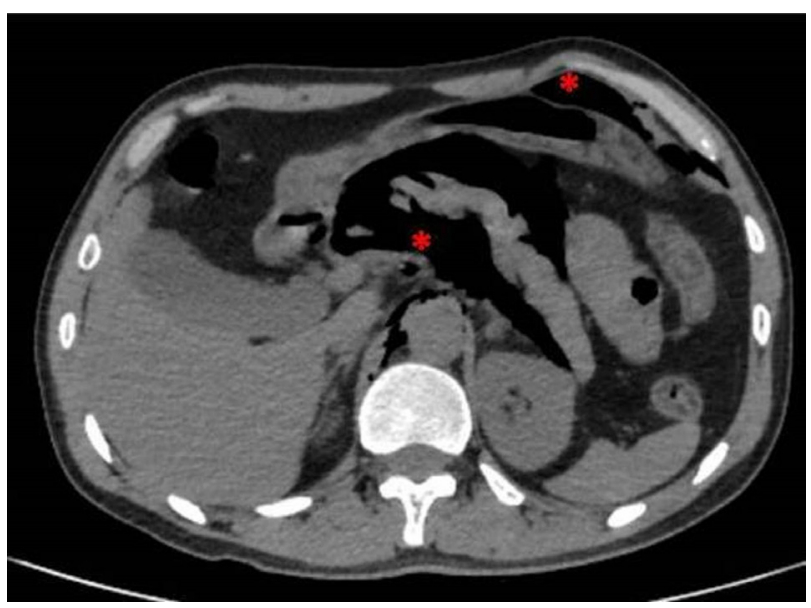

Figure 3. Non-contrast enhanced axial sections through upper abdomen shows significant air collection around pancreas suggestive of pneumoretroperitoneum and within the peritoneal cavity (asterisk).

Unfortunately, the patient could not be revived and succumbed.

\section{Discussion}

Air is normally present within certain locations in the body, such as para-nasal sinuses and aero-digestive tract. Presence of air in locations such as visceral spaces of head and neck, mediastinum and retroperitoneum indicate a pathological process such as trauma. Often, the ectopic air reaches to locations far from the site of primary pathology through the anatomical planes and boundaries. Hence, an in-depth knowledge of connections between various compartments facilitates the quick detection of the cause, especially if no local site injury is identified.

The connection between the neck and chest is through (1):

- Pretracheal space: It is the space between trachea, the strap muscles and pretracheal fascia. The thyroid cartilage in the neck, pericardium and pleura at level of carina in mediastinum form the upper and lower boundaries of this space.

- Visceral space: It contains thyroid and parathyroid glands, trachea, oesophagus, hypopharynx and laryngopharynx. This space communicates with 
mediastinum, allowing free movement of air/fluid (2).

- Carotid space: This space has contribution from all three layers of cervical fascia forming the carotid sheath and contains the carotid artery, internal jugular vein, and the vagus nerve.

- Retropharyngeal space: Anteriorly, it is bounded by buccopharyngeal fascia and posteriorly, by alar fascia. It extends from the skull base to T4 vertebral body. Posterior to the retropharyngeal space is the danger space which a potential space is bounded by alar fascia anteriorly and prevertebral fascia posteriorly. The retropharyngeal space contains fat and retropharyngeal group of lymph nodes (medial and lateral).

- Perivertebral space: This space extends from the skull base to coccyx and is bounded anteriorly by the prevertebral fascia. It contains muscles, vertebral artery and vein, spinal nerves.

There is direct communication between the visceral and retropharyngeal spaces with the mediastinum, allowing free movement of air/fluid between the two compartments (2).

There are three defects in the diaphragmatic attachments which connect the endothoracic fascia (covering the superior diaphragmatic surface) and fascia transversalis (covering inferior diaphragmatic surface) anteriorly (1):

(a) One defect in the midline, between the slips of sternal origin of the diaphragm arising from the xiphoid process and (b) two parasagittal defects between the sternal and costal origins of the diaphragm - the foramen of Morgagni, through which traverses the internal mammary vessels that continue in the abdomen as the superior epigastric vessels (1). The parietal pleura is connected to the endothoracic fascia (2-4). Air/fluid enters the space between the transversalis fascia and the parietal peritoneum, the preperitoneal space, laterally to the flank, and the retroperitoneum through these diaphragmatic defects (3).

Posteriorly, the endothoracic and the transversalis fascia blend behind the diaphragm, and the aortic and esophageal hiatus, resulting in communication between mediastinum and retroperitoneal compartments $(1,2)$. Other focal defects in the tendinous part of the diaphragm or the certain rare entities like septum transversum lacunar aplasia, may also allow transdiaphragmatic migration of air $(4,5)$.

Few cases have demonstrated these anatomical communications. Allard et al (6), reported a case of pneumorachis and pneumocephalus after blunt trauma to thorax without any evidence of spinal or skull fractures. Anbarasu and Khajanchi (7) in 2018 reported a case of pneumocephalus in blunt thoracic trauma where the involved mechanism of migration of air was along the carotid sheaths to the cranial cavity.

\section{Conclusion}

Present case shows migration of air through three compartments, i.e., neck, chest and abdomen, reaching far away from the primary site of injury, i.e., the base of skull. In cases where local site does not reveal any cause of ectopic air, it is always important to review potential sites for injury, based on these anatomical communications, which can assist in timely patient management.

\section{Authors' contributions}

Conception: GS, AS, SV. Acquisition of data: GS and SV. Analysis and/or interpretation of data: GS, AS, SV. Drafting the manuscript: GS. Revising the manuscript critically for important intellectual content: GS, AS, SV. Approval of the version of the manuscript to be published: GS, AS, SV.

\section{Ethical issues}

Informed consent was taken from patient's next of kin.

\section{References}

1. Frias Vilaça A, Reis AM, Vidal IM. The anatomical compartments and their connections as demonstrated by ectopic air. Insights Imaging 2013; 4(6): 759-72. doi: 10.1007/s13244-013-0278-0.

2. Maunder RJ, Pierson DJ, Hudson LD. Subcutaneous and mediastinal emphysema. Pathophysiology, diagnosis, and management. Arch Intern Med 1984; 144(7): 1447-53.

3. Roncati L, Pusiol T, Scialpi M. The endothoracic fascia: an anatomic site in which primary liposarcoma may arise. Lung 2015; 193(6): 1055-6. doi: 10.1007/s00408-015-97983.

4. Kleinman PK, Brill PW, Whalen JP. Anterior pathway for transdiaphragmatic extension of pneumomediastinum. AJR Am J Roentgenol 1978; 131(2): 271-5. doi: 10.2214/ ajr.131.2.271.

5. Lidid L, Valenzuela J, Villarroel C, Alegria J. Crossing the barrier: when the diaphragm is not a limit. AJR Am J Roentgenol 2013; 200(1): W62-70. doi: 10.2214/ajr.11.8264.

6. Allard E, Selim J, Veber B. Pneumocephalus and pneumorachis after blunt chest trauma without spinal fractures: a case report. J Med Case Rep 2019; 13(1): 317. doi: 10.1186/s13256-019-2208-3.

7. Anbarasu A, Khajanchi M. Pneumocephalus following thoracic trauma: a rare entity along the carotid sheath. ANZ J Surg 2018; 88(4): 382-3. doi: 10.1111/ans.13388. 